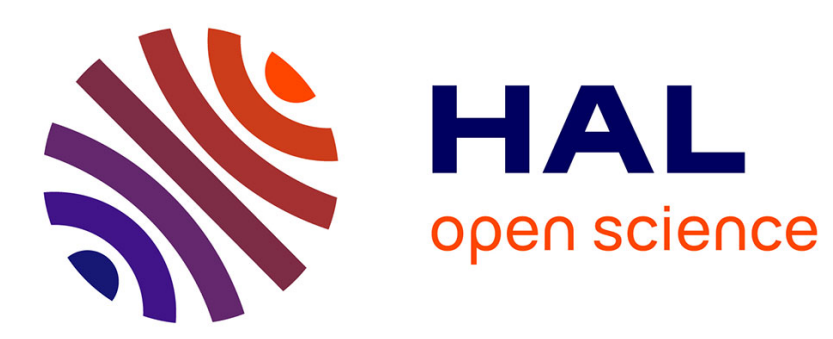

\title{
A Mixed Surface Volume Integral Formulation for the Modeling of High Frequency Coreless Inductors
}

Zacharie de Greve, Jonathan Siau, Gérard Meunier, Jean-Michel Guichon, Olivier Chadebec

\section{- To cite this version:}

Zacharie de Greve, Jonathan Siau, Gérard Meunier, Jean-Michel Guichon, Olivier Chadebec. A Mixed Surface Volume Integral Formulation for the Modeling of High Frequency Coreless Inductors. IEEE Transactions on Magnetics, 2016, 52 (3), 10.1109/TMAG.2015.2497004 . hal-01385601

\section{HAL Id: hal-01385601 https://hal.science/hal-01385601}

Submitted on 16 Nov 2020

HAL is a multi-disciplinary open access archive for the deposit and dissemination of scientific research documents, whether they are published or not. The documents may come from teaching and research institutions in France or abroad, or from public or private research centers.
L'archive ouverte pluridisciplinaire HAL, est destinée au dépôt et à la diffusion de documents scientifiques de niveau recherche, publiés ou non, émanant des établissements d'enseignement et de recherche français ou étrangers, des laboratoires publics ou privés. 


\title{
A Mixed Surface Volume Integral Formulation for the Modeling of High Frequency Coreless Inductors
}

\author{
Zacharie De Grève ${ }^{4}$, Jonathan Siau ${ }^{1,2}$, Gérard Meunier ${ }^{1,3}$, Jean-Michel Guichon ${ }^{1}$ and Olivier Chadebec ${ }^{1,3}$ \\ ${ }^{1}$ University of Grenoble Alpes, G2ELAB, F-38000 Grenoble, France \\ ${ }^{2}$ CEDRAT S.A., F-38000 Grenoble, France \\ ${ }^{3} \mathrm{CNRS}, \mathrm{G} 2 \mathrm{ELAB}, \mathrm{F}-38000$ Grenoble, France \\ ${ }^{4}$ Electrical Power Engineering Unit, University of Mons, Bd Dolez, 31, B-7000 Mons, Belgium
}

\begin{abstract}
An original integral formulation dedicated to the high frequency modeling of electromagnetic systems without magnetic materials is presented. The total current density (i.e. conduction plus displacement currents) is approached by facet elements so that resistive, inductive and capacitive effects are all modeled. The method avoids moreover the volume mesh of the conductors, which is too dense at high frequencies, due to the skin and proximity effects appearing $e$.g. in wound inductors. Surface impedance boundary conditions are employed to that end. The formulation is general and suitable for non simply connected domains. It is first compared with the finite element method on an academic test case, and is then experimentally validated on a coreless wound inductor, using an impedance analyzer.
\end{abstract}

Index Terms-Computational electromagnetics, Integral equations, Electromagnetic compatibility

\section{INTRODUCTION}

C URRENT progresses in power electronics are enabling the use of higher switching frequencies, up to several $\mathrm{MHz}$ [1]. This permits to reduce the volume and the mass of the passive components, which is critical for transportation applications. This frequency range is also used for the design of wireless power transfer systems [2] (wireless battery charging [3], transcutaneous energy transmitters [4], etc.). At such frequencies, parasitic capacitive effects cannot be neglected, as they may induce ElectroMagnetic Compatibility (EMC) problems, or influence the resonant frequency of a wireless power transmitter. These effects are moreover strongly influenced by the geometry of the wound component (i.e. the position of the wires in the winding window). Appropriate numerical tools, allowing broadband analyses with acceptable computational costs, need therefore to be proposed to the engineering community.

Several attempts have been made in the literature. In [5], a full wave Finite Element (FE) formulation, modified in order to enhance numerical stability at low frequencies, has been proposed. A FE method requires however a full mesh of the geometry, including the air. Such a mesh may become very large, and is moreover not easy to obtain in 3D, particularly for wound components, where structured and unstructured meshes may be present simultaneously. A broadband integral formulation, which is based on a volumetric loop-star decomposition, is presented in [6]. It does not need a mesh of the air, but requires a volume mesh of the active materials. Moreover, it does not provide a direct circuit interpretation of the field equations, which is an important asset when modeling the physical component in its direct electrical environment. Reference [7]

\footnotetext{
Manuscript received xxx, revised xxx. Corresponding author: Z. De Grève (email: zacharie.degreve@umons.ac.be).

Color versions of one or more of the figures in this paper are available online at http://ieeexplore.ieee.org.

Digital Object Identifier xx.xxxx/TMAG.xxxx.xxxxxxx
}

establishes direct links between FE formulations and circuit representations, but suffers from the drawback associated to a FE approach.

The Partial Element Equivalent Circuit (PEEC) method, initially proposed for high frequency problems including time retardation, offers a framework for the efficient modeling of such physical phenomena [8], [9]. It is an integral method which associates an RLC circuit to a meshed geometry, and which does not require the mesh of the air. An adaptation to unstructured meshes, based on the use of facet elements, has recently been proposed in the pure magnetodynamic case [10]. This approach requires a full volume mesh of the conductors. However, the skin and proximity effects, which appear in the conductors at such frequencies, lead to very fine meshes. Therefore, in this paper, the formulation of [10] is adapted so as to include Surface Impedance Boundary Conditions (SIBCs [11]). By doing so, the volume mesh of the conductors is avoided. The capacitive effects are moreover taken into account using the formalism presented in [12].

The original formulation is first described in the pure magnetodynamic case in Section II, and is compared with the Finite Element Method (FEM) on an torus submitted to an external uniform magnetic flux density aligned with its main axis. A particular attention is paid to the modeling of the eddy current losses. The formulation is then extended to take capacitive effects into account in Section III, and is experimentally validated on a coreless wound inductor, characterized by an impedance analyzer.

\section{Magnetodynamic formulation using Surface Impedance BOUNDARY CONDITIONS}

\section{A. Formulation}

The pure magnetodynamic formulation is presented in this section. The magnetic vector and electric scalar potentials, A and $V$ respectively, are first expressed in their integral form: 


$$
\begin{aligned}
\mathbf{A}(\mathbf{x}) & =\frac{\mu_{0}}{4 \pi}\left(\int_{\Omega_{J}} \frac{\mathbf{J}}{r} \mathrm{~d} \Omega\right), \\
j \omega V(\mathbf{x}) & =\frac{1}{4 \pi \epsilon_{0}}\left(\int_{\Omega_{J}} \mathbf{J g r a d} \frac{1}{r} \mathrm{~d} \Omega\right),
\end{aligned}
$$

in the frequency domain, with $j$ the imaginary number, $\omega$ the angular frequency, $\mathbf{J}$ the conduction current density, $\mu_{0}$ the magnetic permeability of vacuum, $r$ the distance between the field sources and point $\mathbf{x}$. The integrals are defined on $\Omega_{J}$, namely the domain containing the massive conductors. Scalar and vector fields are complex quantities in this paper.

The potentials are injected into the electric field $\mathbf{E}$ equation:

$$
\mathbf{E}+j \omega \mathbf{A}+\operatorname{grad} V=0
$$

A Galerkin procedure is then applied on eq. (3), by using the current density $\mathbf{J}=\sigma \mathbf{E}$, with $\sigma$ the electrical conductivity, as the problem unknown. First order facet elements defined on $\Omega_{J}$ are employed:

$$
\left.\mathbf{J}\right|_{\Omega_{J}}=\sum_{i \in \mathcal{F}_{J}} \mathbf{w}_{i} I_{i}
$$

where $\mathbf{w}_{i}$ is a volume facet basis function (in $\left[\mathrm{m}^{-2}\right]$ ), and $I_{i}$ the current crossing the facet $i \in \mathcal{F}_{J}$ (in [A], with $\mathcal{F}_{J}$ the set of facets in domain $\Omega_{J}$ ). By doing so, the field equations are translated into circuit equations supported by the dual mesh [10]. This leads to the resolution of the following system of circuit equations:

$$
([R]+j \omega[L])\{\mathbf{I}\}=\{\Delta V\},
$$

with $\{\mathbf{I}\}$ the branch currents crossing the facets, and $\{\Delta V\}$ the branch voltages. $[R]$ is a sparse finite element resistance matrix, and $[L]$ the full inductance matrix [10]:

$$
R_{i j}=\int_{\Omega_{J}} \frac{\mathbf{w}_{i} \mathbf{w}_{j}}{\sigma} \mathrm{d} \Omega_{J}, \quad L_{i j}=\frac{\mu_{0}}{4 \pi} \int_{\Omega_{J}} \mathbf{w}_{i} \int_{\Omega_{J}} \frac{\mathbf{w}_{j}}{r} \mathrm{~d} \Omega_{J}^{2},
$$

with $i, j \in \mathcal{F}_{J}$. However, at high frequencies, the skin and proximity effects in the massive conductors lead to very fine meshes of $\Omega_{J}$, which drastically increases the size of matrixes $[R]$ and $[L]$. Therefore, in this paper, the formulation (5) is extended so as to avoid the volumic mesh of the conductors.

To that end, the current density $\mathbf{J}$ is expressed on the boundary $\Gamma_{J}$ of $\Omega_{J}$ :

$$
\left.\mathbf{J}\right|_{\Gamma_{J}}=\sum_{i \in \mathcal{E}_{J}} \mathbf{w}_{S, i} I_{S, i}
$$

with $\mathbf{w}_{S, i}$ the surface facet basis functions (in $\left[\mathrm{m}^{-1}\right]$ ), $\mathcal{E}_{J}$ the set of edges of $\Gamma_{J}$, and $I_{S, i}$ the line current crossing an edge $i$ (in $\left[\mathrm{Am}^{-1}\right]$, see Figure 1).

The total current $I_{i}$ in the skin layer (i.e. the current crossing the surface $S_{i}$ in $\Omega_{J}$, supported by the edge $i$ of $\Gamma_{J}$ ) needs to be expressed as a function of the edge currents $I_{S, i}$. To that end, a first order Leontovitch SIBC is employed [11]: the air-conductor interface is approached by a semi-infinite plane excited by tangential fields. By solving the Maxwell equations

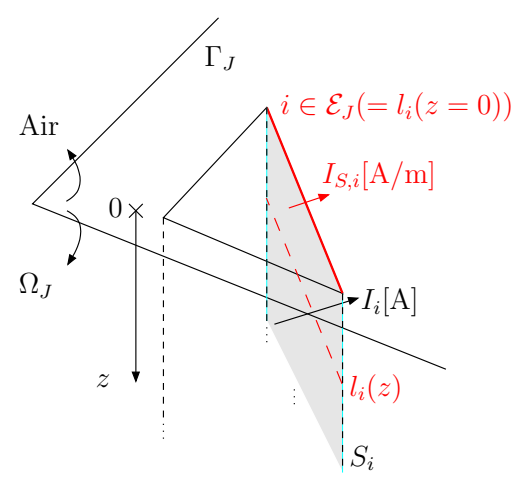

Fig. 1. Focus on an element of $\Gamma_{J}$.

on this simplified case, it is shown that the electrical quantities decay exponentially in the skin depth [11]. We have therefore:

$$
I_{i}=\int_{0}^{\infty} I_{l_{i}(z)} d z=\int_{0}^{\infty} I_{S, i} e^{-\frac{1+j}{\delta} z} d z=\frac{\delta}{1+j} I_{S, i},
$$

with $\delta$ the skin depth and $z$ the distance inside the conductor. The current density $\mathbf{J}$ of equation (7) becomes:

$$
\mathbf{J}=\sum_{i \in \mathcal{E}_{J}} \mathbf{w}_{S, i} \frac{1+j}{\delta} I_{i}
$$

The potentials (1) and (2) are injected in equation (3), which is then projected on $\Gamma_{J}$ with the surface facet basis functions $\mathbf{w}_{S, i}, i \in \mathcal{E}_{J}$ :

$$
\begin{aligned}
\int_{\Gamma_{J}} \mathbf{w}_{S, i} \frac{\mathbf{J}}{\sigma} \mathrm{d} \Gamma+j \omega \frac{\mu_{0}}{4 \pi} \int_{\Gamma_{J}} \mathbf{w}_{S, i} & \left(\int_{\Omega_{J}} \frac{\mathbf{J}}{r} \mathrm{~d} \Omega\right) \mathrm{d} \Gamma \\
& +\int_{\Gamma_{J}} \mathbf{w}_{S, i} \operatorname{grad} V \mathrm{~d} \Gamma=0
\end{aligned}
$$

The volume integral on $\Omega_{J}$ in (10) is approached by:

$$
\int_{\Omega_{J}} \frac{\mathbf{J}}{r} \mathrm{~d} \Omega=\int_{\Gamma_{J}} \int_{0}^{\infty} \frac{\mathbf{J}(z=0)}{r} e^{-\frac{1+j}{\delta} z} \mathrm{~d} z \mathrm{~d} \Gamma \simeq \frac{\delta}{1+j} \int_{\Gamma_{J}} \frac{\mathbf{J}}{r} \mathrm{~d} \Gamma
$$

The last term in equation (10) corresponds moreover to the branch voltages $\Delta V_{i}$ between elements of $\Gamma_{J}$ (Fig. 2). Indeed, by applying the divergence theorem, we have:

$$
\int_{\Gamma_{J}} \mathbf{w}_{S, i} \operatorname{grad} V \mathrm{~d} \Gamma_{J}=-\int_{\partial \Gamma_{J}}\left(\mathbf{w}_{S, i} \cdot \mathbf{n}\right) V \mathrm{~d}\left(\partial \Gamma_{J}\right)+\int_{\Gamma_{J}} \operatorname{div} \mathbf{w}_{S, i} V \mathrm{~d} \Gamma_{J},
$$

with $\partial \Gamma_{J}$ the line boundary of $\Gamma_{J}$, if it exists, and $\mathbf{n}$ its normal. The surface facet basis functions respect the following properties, depending on the element orientation:

$$
\begin{aligned}
\operatorname{div}_{S, i} & = \pm \frac{1}{\mathcal{S}_{i}}, \\
\mathbf{w}_{S, i} . \mathbf{n} & = \pm \frac{1}{\mathcal{L}_{i}},
\end{aligned}
$$

with $\mathcal{S}_{i}$ the surface of the element containing the edge $i$, and $\mathcal{L}_{i}$ the length of edge $i$. 
If $i$ corresponds to an edge inside $\Gamma_{J}$, the first term of the second hand of (12) vanishes. Knowing (13), equation (12) represents therefore the difference between the averaged voltages of the elements sharing the edge $i$. If $i$ corresponds to a border edge, (12) becomes the difference between the averaged voltage on edge $i$ and the averaged voltage on the elements to whom edge $i$ belongs, knowing (13) and (14).

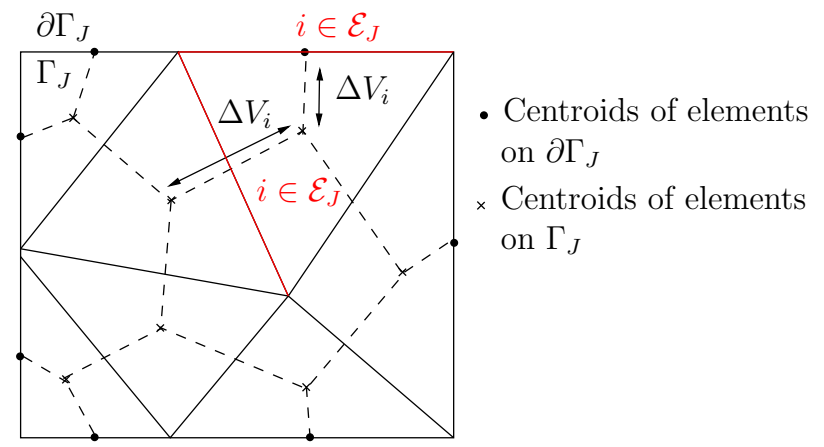

Fig. 2. Primal (plain lines) and dual (dotted lines) meshes of the boundary $\Gamma_{J}$ of the conducting domains modeled using SIBCs.

A system of equations similar to (5) can therefore be written, with the same circuit interpretation. Matrixes $[R]$ and $[L]$ read:

$$
R_{i j}=\int_{\Gamma_{J}} \frac{\mathbf{w}_{S, i} \mathbf{w}_{S, j}}{\sigma *} \mathrm{~d} \Gamma_{J}, \quad L_{i j}=\frac{\mu_{0}}{4 \pi} \int_{\Gamma_{J}} \mathbf{w}_{S, i} \int_{\Gamma_{J}} \frac{\mathbf{w}_{S, j}}{r} \mathrm{~d} \Gamma_{J}^{2},
$$

with $\sigma^{*}=\sigma \delta /(1+j)$ an equivalent complex conductivity. Eddy current losses are given by:

$$
\begin{aligned}
P_{\text {eddy }} & =\int_{\Omega_{J}} \sigma^{-1}|\mathbf{J}|^{2} \mathrm{~d} \Omega_{J}=\int_{\Gamma_{J}} \int_{0}^{\infty} \sigma^{-1}\left|\mathbf{J}(z=0) e^{-\frac{(1+j)}{\delta} z}\right|^{2} \mathrm{~d} z \mathrm{~d} \Gamma \\
& =\int_{\Gamma_{J}} \sigma^{-1}|\mathbf{J}|^{2}\left(\int_{0}^{\infty}\left|e^{-\frac{z}{\delta}} e^{-\frac{j z}{\delta}}\right|^{2} \mathrm{~d} z\right) \mathrm{d} \Gamma=\frac{1}{2} \int_{\Gamma_{J}} \sigma^{-1}|\mathbf{J}|^{2} \delta \mathrm{d} \Gamma
\end{aligned}
$$

\section{B. Test case}

The method is validated on a $0.1 \mathrm{~m}$ diameter torus, made of $0.02 \mathrm{~m}$ copper wire, submitted to an external magnetic flux density aligned with its main axis (see left of Figure 3). A 2D axisymmetric finite element model has been used as a reference. Figure 3 on the right depicts the eddy current losses in the torus as a function of the frequency, for the two models (namely the 2D FEM reference and the PEEC surface formulation). A good agreement is observed betwen the two models (less than $3.5 \%$ relative error), which validates the proposed formulation. The surface PEEC model generates 1153 unknowns (or independent loops), which can be compared to 25921 unknowns for the PEEC volume formulation of [10].

\section{Adding the capacitive efFects}

\section{A. Formulation}

The formulation of the previous section is here extended so that capacitive effects are taken into account, based on the approach presented in [12]. The magnetic vector and electric scalar potentials are first re-written:

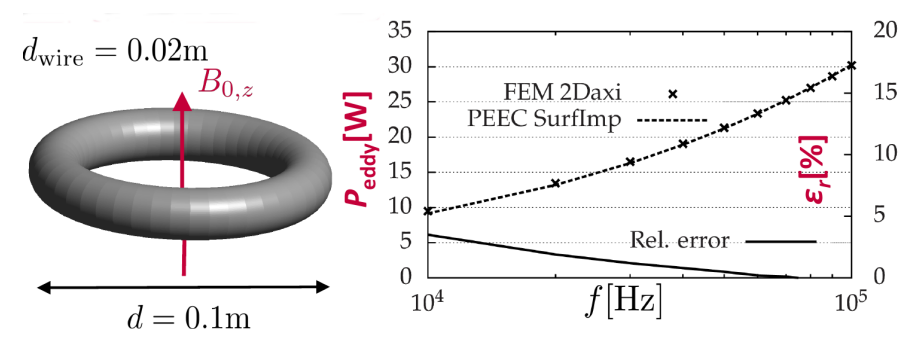

Fig. 3. Torus submitted to an external magnetic flux density (left), and eddy current losses in the torus, obtained using a 2D FE model and the proposed PEEC formulation.

$$
\begin{aligned}
\mathbf{A}(\mathbf{x}) & =\frac{\mu_{0}}{4 \pi}\left(\int_{\Omega_{J}} \frac{\mathbf{J}}{r} \mathrm{~d} \Omega+\int_{\Omega_{D}} j \omega \frac{\mathbf{P}}{r} \mathrm{~d} \Omega\right), \\
j \omega V(\mathbf{x}) & =\frac{1}{4 \pi \epsilon_{0}}\left(\int_{\Omega_{J}} \mathbf{J g r a d} \frac{1}{r} \mathrm{~d} \Omega+\int_{\Omega_{D}} j \omega \mathbf{P g r a d} \frac{1}{r} \mathrm{~d} \Omega\right),
\end{aligned}
$$

with $\mathbf{P}$ the electric polarization, $\epsilon_{0}$ the electric permittivity of vacuum, and $\Omega_{D}$ the domain containing the dielectrics.

The total current density $\mathbf{J}_{t}$ is taken here as the unknown:

$$
\mathbf{J}_{t}=\mathbf{J}+j \omega \mathbf{D}=\mathbf{J}+\epsilon j \omega \mathbf{E}=(\sigma+j \omega \epsilon) \mathbf{E},
$$

with D the electric flux density. First order volume facet elements defined on $\Omega=\Omega_{J} \cup \Omega_{D}$ are employed, but compared to [12], the conduction current density $\mathbf{J}$ is expressed on $\Gamma_{J}$ instead of $\Omega_{J}$, using a first order SIBC. Volume elements are on the other hand employed in $\Omega_{D}$. By assuming that $\sigma=0$ in $\Omega_{D}$ and $\sigma>>\epsilon$ in $\Omega_{J}$, we have therefore:

$$
\mathbf{J}_{t}=\underbrace{\left.\mathbf{J}_{t}\right|_{\Omega_{D}}}_{=j \omega \mathbf{D}}+\underbrace{\left.\mathbf{J}_{t}\right|_{\Gamma_{J}}}_{=\mathbf{J}}=\sum_{k \in \mathcal{F}_{D}} \mathbf{w}_{k} I_{k}+\sum_{i \in \mathcal{E}_{J}} \mathbf{w}_{S, i} \frac{1+j}{\delta} I_{i},
$$

with $\mathcal{F}_{D}$ the set of facets of domain $\Omega_{D}$. Again, a Galerkin procedure is applied on equation (3), but with the expressions (17) and (18) for the potentials, and with the discretization (20) for $\mathbf{J}_{t}$. The following system of equation is obtained [12]:

$$
\left([R]+j \omega[L]+\frac{1}{j \omega}([C]+[P])\right)\{\mathbf{I}\}=\{\Delta V\}
$$

As explained in [12], [C] is a sparse FE matrix which models capacitive effects inside the domain $\Omega_{D}$. Matrix $[P]$ is dense, and represents capacitive interactions outside domain $\Omega_{D}$. It is defined by adding capacitive branches between the facets belonging to the external border $\Gamma_{\text {ext }}$ and an external reference node $V_{\infty}$. Figure 4 shows for instance the cross section of a winding wire: a volume mesh is depicted for the insulation surrounding the wire, whereas the conducting part is not meshed.

The matrixes $[C]$ and $[P]$ remain unchanged compared to [12]. On the other hand, it can easily be shown that matrixes $[R]$ and $[L]$ are similar to equations (15), except for the surfacevolume coupling terms. Indeed, if $i \in \mathcal{E}_{J}$ and $j \in \mathcal{F}_{D}$, the inductive terms read:

$$
L_{i j}=\frac{\mu_{0}}{4 \pi} \int_{\Gamma_{J}} \mathbf{w}_{S, i}\left(\int_{\Omega_{D}} \frac{\epsilon-\epsilon_{0}}{\epsilon} \frac{\mathbf{w}_{j}}{r} \mathrm{~d} \Omega\right) \mathrm{d} \Gamma,
$$




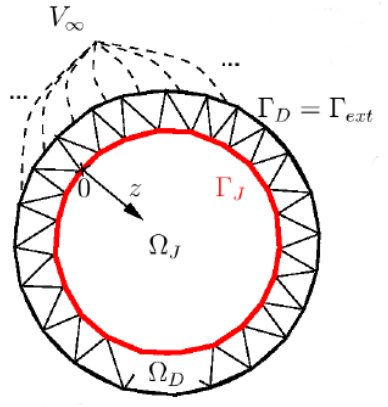

Fig. 4. Mesh of the cross section of a winding wire. A volume mesh is observed for the insulation layer surrounding the wire, xhereas the conducting part is not meshed.

with $\epsilon$ the electric permittivity of the dielectric.

\section{B. Experimental validation}

The capacitive formulation is tested on a 19-turn, 2 layer coreless wound inductor, with conductors of radius $0.1 \mathrm{~mm}$ surrounded by an insulating layer of $0.0215 \mathrm{~mm}$, with $\epsilon_{r}=2.5$. The coilformer is characterized by an $\epsilon_{r}=3$. The radius of the former is equal to $12.5 \mathrm{~mm}$. A circuit solver has been employed to solve the system (21), leading to 18735 unknowns (or independent circuit loops). This has to be compared to the 52000 unknowns of the full volumic formulation [12], which shows the interest of the proposed method.

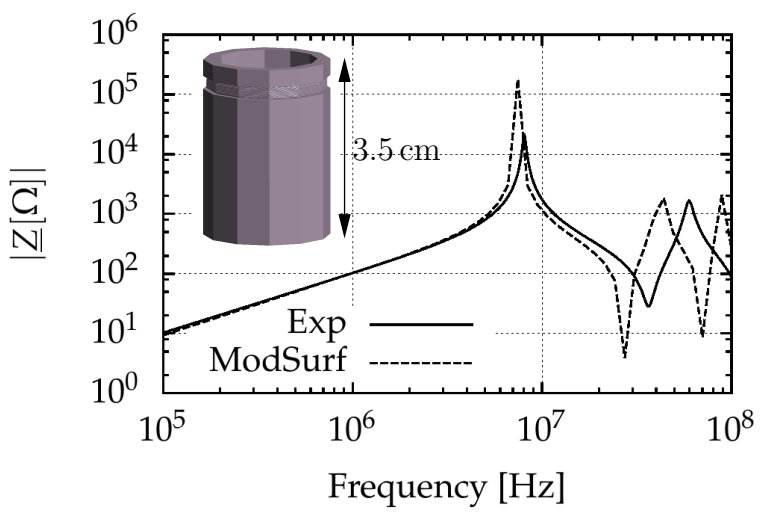

Fig. 5. Impedance of the test coil, measurement and capacitive model.

An impedance analyzer Agilent 4294A has been used to measure the impedance of the component. Figure 5 depicts the module of the impedance of the test coil, in the case of the measurement (plain lines) and the model (dotted lines). One can see that the position of the first resonance is quite similar for the two curves, which is less valid for the higher order resonances. This can be associated to uncertainties regarding the parameters of the test coil (geometry as well as material parameters). Indeed, it is difficult to know exactly the value of the relative permittivity of the insulation surrounding the wires (manufacturer catalogs give usually a tolerance of $+-30 \%$ on that value, and it is sometimes even not available). The position of the wires in the winding window influences moreover greatly the parasitic capacitances, and these positions are usually not known accurately. Nevertheless, the resonance/anti-resonance patterns of the measured impedance curve are well reproduced by our model.

Moreover, the amplitude of the peaks appears to be higher with the model, which may be due to an inaccurate estimation of the coil resistance with the first order SIBC. Higher order SIBCs will be investigated in a future work.

\section{Conclusion}

An unstructured PEEC formulation which takes capacitive effects into account, and make use of Surface Impedance Boundary Conditions in the conductors, has been proposed in this paper. The formulation is valid for non simply connected domains. A volume mesh of the dielectrics is required, whereas a surface mesh of the conductors is sufficient. By doing so, dense meshes, which are needed at high frequencies due to the skin and proximity effects in the conductors, are avoided. A numerical validation of the Surface Impedance formulation has first been proposed on a torus in pure magnetodynamics, by comparing with the FEM. The capacitive formulation has then been tested on a 19 turn coreless inductor, and compared with experimental data obtained using an impedance analyzer. A good agreement with measurements was obtained. The observed deviations can be attributed to uncertainties related to wound components (geometry as well as material characteristics). This will be studied in a future work. The method will also be extended to higher order SIBCs. A full surface formulation (i.e. which relies on a surface mesh of the dielectrics as well) will also be proposed.

\section{REFERENCES}

[1] O. Deblecker, C. Versèle, Z. De Grève, "SiC vs. Si-based isolated DC-DC converters: assesment of power loss and mass gains using a multiobjective optimization tool", Int. Review of Elec. Eng., 9(4), pp.708-716, 2014.

[2] S.Y.R. Hui, W. Zhong and C.K. Lee, "A critical review on recent progress in mid-range wireless power transfer", IEEE Trans. Power Electronics, 29(9), pp. 4500-4511, 2014.

[3] B. Choi, J. Nho et al, "Design and implementation of low-profile contactless battery charger using planar printed circuit board windings as energy transfer device", IEEE Trans. Ind. Electron., 51(1), pp. 140-147, 2004.

[4] D.W. Ferreira, "Topics in design and analysis of transcutaneous energy transfer to ventricular assist devices", PhD Thesis, Univ. Sao Paulo, 2013.

[5] R. Hipmair, F. Krämer and J. Ostrowski, "A Robust Maxwell formulation for all frequencies”, IEEE Trans. Mag., 44(6), pp. 682-685, 2008.

[6] G. Rubinacci and A. Tamburrino, "A broadband volume integral formulation based on edge-elements for full-wave analysis of lossy interconnects", IEEE Trans. Antennas and Propagation, 54(10), pp. 2977-2989, 2006.

[7] A. Demenko and J. Sykulski, "Magneto-electric network models in electromagnetism", COMPEL-The Int. Jour. for Computation and Mathematics in Electrical and Electronic Engineering, 25(3), pp. 581-588, 2006.

[8] A. Ruelhi, "Equivalent circuit models for three-dimensional multiconductor systems", IEEE Trans. Micro. th. and tech., 22(3), pp. 216-221, 1974.

[9] J.Ekman, Electromagnetic Modeling Using the Partial Element Equivalent Circuit Method, PhD Thesis, Lulea Univ. of Technology, Sweden, 2003. Available http://staff.www.ltu.se/ jekman/Pres/PhDThesis.pdf

[10] T.T. Nguyen, G. Meunier, J.-M. Guichon, O. Chadebec and T.-S. Nguyen "An integral formulation for the computation of 3D eddy current using facet elements", IEEE Trans. Mag., 50(2), pp. 549-552, 2014

[11] S. Yuferev, N. Ida, "Selection of the surface impedance boundary condition for a given problem", IEEE Trans. Mag., 35(3), pp.1486-1489, 1999.

[12] J. Siau, G. Meunier, O. Chadebec, J.-M. Guichon, R. Perrin-Bit, "Volume integral formulation using facet elements for electromagnetic problems considering conductors and dielectrics", in Proc. of the Int. Compumag Conf., Montreal, 2015. 